\title{
Message from the President, Health Informatics Society (HISSL) of Sri Lanka
}

Sri Lanka Journal of Bio-Medical Informatics 2009;1(1): 3

DOI: $10.4038 /$ sljbmi.v1i1.1479

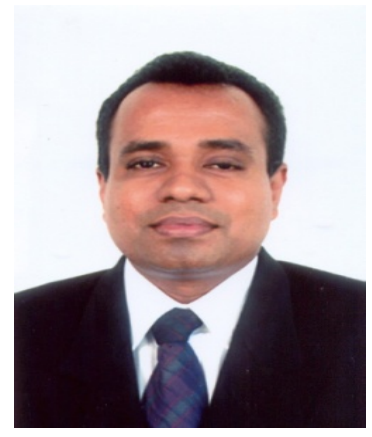

It is with great pleasure that I send this message to the Inaugural Issue of the Sri Lanka Journal of Biomedical Informatics (SLJBMI). The SLJBMI is a joint effort between the Specialty Board in Biomedical Informatics of the Postgraduate Institute of Medicine (PGIM), University of Colombo, Sri Lanka and, the Health Informatics Society of Sri Lanka (HISSL).

The Health Sector in Sri Lanka is undergoing rapid change due to the implementation of various e-Health initiatives in many health care institutions in different parts of the country. These initiatives largely remain unknown to many. We hope that the Current Practice section of the SLJBMI would serve as a sounding board for these initiatives and make them widely known.

The commencement of the M.Sc Course in Bio-Medical Informatics at the PGIM is the first step towards developing the specialty as an academic discipline in Sri Lanka. This would catalyze research in the field in Sri Lanka. SLJBMI would also serve as a vehicle for publishing this research. The academic focus of the Journal therefore would grow in the years to come.

The idea for the SLJBMI was conceived about six months ago. It had a short gestation period and a smooth delivery thanks largely to the efforts of its Editor-in-Chief, Dr. B.J.C. Perera. I wish to thank him for all his efforts.

I hope that the SLJBMI would grow from strength to strength.

\section{Dr. Vajira H. W. Dissanayake MBBS, PhD}

President, Health Informatics Society of Sri Lanka

Chairperson, Specialty Board in Biomedical Informatics, Postgraduate Institute of Medicine, University of Colombo, Sri Lanka

Senior Lecturer and Head of Department of Anatomy, Faculty of Medicine, University of Colombo, Sri Lanka 